\title{
MicroRNAs in the aqueous humor of patients with different types of glaucoma
}

\author{
Ewa Kosior-Jarecka ${ }^{1} \cdot$ Marcin Czop $^{2} \cdot K^{2}$ arolina Gasińska ${ }^{1}$ [D $\cdot$ Dominika Wróbel-Dudzińska $^{1} \cdot$ Daniel P. Zalewski $^{3}$. \\ Anna Bogucka-Kocka ${ }^{3} \cdot$ Janusz Kocki ${ }^{2} \cdot$ Tomasz Żarnowski $^{1}$
}

Received: 4 November 2020 / Revised: 16 April 2021 / Accepted: 19 April 2021 / Published online: 30 April 2021

(c) The Author(s) 2021

\begin{abstract}
Purpose The aim of the study was to compare the frequency and the level of expression of selected miRNAs in the aqueous humor of patients with various types of glaucoma.

Methods The studied group consisted of 42 patients with glaucoma: 19 with primary open-angle glaucoma (POAG), 14 with pseudoexfoliation glaucoma (PEXG), 9 with primary angle closure glaucoma (PACG), and the control group of 36 patients with senile cataract without glaucoma. The real-time polymerase chain reaction method was used to analyze the expression of miRNAs. Results There were no significant differences in the frequency and the level of miRNA expression between various types of glaucoma. There was a tendency for hsa-miR-6722-3p and hsa-miR-184 to be expressed more frequently in PEXG and hsa-miR-1260b in POAG. The expression levels of hsa-miR-1260b and hsa-miR-6515-3p were correlated with age in POAG. Target annotation and functional analyses showed that genes targeted by the most frequently expressed miRNAs (hsa-miR-1202, -1260b, -184, -187-5p, -6515-3p, -6722-3p, and hsa-mir-4634) are involved mainly in response to hypoxia, cardiovascular system development, and apoptosis.

Conclusion Hsa-miR-1260b was the most abundantly expressed among studied miRNAs and may be a potential biomarker of clinical status in PEXG and PACG.
\end{abstract}

Keywords MicroRNA $\cdot$ Aqueous humor $\cdot$ Glaucoma $\cdot$ POAG $\cdot$ PACG $\cdot$ PEXG

\section{Key messages}

- While glaucoma is the second leading cause of blindness worldwide, the relationship between expression patterns of microRNA (miRNA) and glaucoma pathogenesis is little known.

- Individual miRNAs profiles vary for patients with different types of glaucoma.

- Hsa-miR-1260b may be a potential biomarker of clinical status in PEXG and PACG

- Further studies in this area may drive the discovery of new insights into the genetic aspects of glaucoma.

Ewa Kosior-Jarecka and Marcin Czop authors have equal authorship

Anna Bogucka-Kocka, Janusz Kocki and Tomasz Żarnowski all three authors have equal senior authorship

Karolina Gasińska

karolina.gasinska@onet.eu

1 Department of Diagnostics and Microsurgery of Glaucoma, Medical University of Lublin, ul. Chmielna 1,

20-079 Lublin, Poland
2 Department of Clinical Genetics, Medical University of Lublin, ul. Radziwiłłowska 11, 20-080 Lublin, Poland

3 Department of Biology and Genetics, Medical University of Lublin, ul. Chodźki 4a, 20-093 Lublin, Poland 


\section{Introduction}

Glaucoma is a multifactorial disease involving retinal ganglion cells and has been estimated by WHO as the second leading cause of blindness worldwide [1]. The main risk factor for glaucoma is increase in intraocular pressure (IOP) caused by decreased outflow of aqueous humour $(\mathrm{AH})$ from the anterior chamber [2]. Glaucoma is a group of optic neuropathies with similar morphology of the visual field defect and optic disc appearance. However, the mechanisms underlying the disease might be various. In most cases, the disease is connected with elevated IOP, but there are also cases with continuous progression despite low IOP values. Additionally, the mechanisms causing IOP elevation are also different in glaucoma types. MiRNAs are small, noncoding RNA molecules involved in RNA silencing and regulation of gene expression at the posttranscriptional level. MiRNAs predominantly act to reduce target gene expression [3]. Expression of miRNAs is often typical for a particular tissue or during essential cellular processes $[4,5]$. MiRNAs act intracellularly but have also been detected in most body fluids, where they are preserved in microvesicles, exosomes, or bound to carrier proteins, providing a remarkable stability of miRNA [6]. A lot of extracellular miRNAs from biofluids has been identified as biomarkers for cancer, cardiovascular diseases, diabetes, ocular diseases, and many other disorders [4, 7-14]. Ocular fluids (tears, $\mathrm{AH}$ and vitreous humour) also have been reported to contain extracellular miRNAs [4, 15-18]. Alterations in levels of AH components, including cells, proteins, and miRNAs, may reflect the pathogenic process underlying the increase in IOP during the course of glaucoma. Only a few studies investigated miRNA expression in $\mathrm{AH}$ of glaucoma patients $[4,6,15]$. Therefore, it is interesting whether the expression patterns of microRNA (miRNA) may reflect this variety. Tanaka et al. [19] showed that individual miRNA profiles vary for patients with glaucoma and the number of commonly detected miRNAs was limited, but combining these markers had the potential to increase the sensitivity of glaucoma diagnosis and to predict stage of advancement $[4,19]$, which is not possible with genome analysis [19].

The aim of the study was to compare the frequency and the levels of selected miRNAs expression in $\mathrm{AH}$ of patients with various types of glaucoma: primary open angle glaucoma (POAG), pseudoexfoliation glaucoma (PEXG), and primary angle closure glaucoma (PACG) as well as to identify miRNA-dependent mechanisms contributing to these types of glaucoma.

\section{Materials and methods}

The study was performed in accordance with the tenets of the Declaration of Helsinki and the study design was approved by the Ethics Committee at the Medical University of Lublin (approval No. KE-0254/107/2020). The studied group consisted of 42 patients with glaucoma (19 POAG, 14 PEXG, and 9 PACG) and 36 control patients with senile cataract without glaucoma nor elevated IOP. Informed consent was signed by all participants before enrolment to the study. Patients' demographic and clinical data are presented in Table 1.

All participants underwent planned cataract surgery at the Department of Diagnostics and Microsurgery of Glaucoma, Medical University of Lublin, Poland.

Before the surgery, all patients underwent detailed ophthalmic examination, included best corrected visual acuity (BCVA) assessment (using Snellen charts), slit-lamp biomicroscopy, gonioscopy, ultrasonic central corneal thickness measurements, IOP with Goldmann applanation tonometry, and stereoscopic optic nerve head (ONH) examination as well as a detailed medical history analysis. Detailed inclusion criteria were as follows:

1. Signed informed consent

2. The age of over 18 years old

Table 1 Demographic and clinical characteristics of the studied groups

\begin{tabular}{|c|c|c|c|c|c|}
\hline \multirow[t]{2}{*}{ Feature } & \multicolumn{4}{|l|}{ Studied groups } & \multirow[t]{2}{*}{ Statistical analysis } \\
\hline & PACG $(n=9)$ & POAG $(n=19)$ & PEXG $(n=14)$ & Cataract $(n=36)$ & \\
\hline \multicolumn{6}{|l|}{ Gender: } \\
\hline Male & 6 & 8 & 6 & 17 & $p>0.05$ \\
\hline Female & 3 & 11 & 8 & 19 & \\
\hline Age $^{1}$ & $75.00 \pm 8.40^{\mathrm{a}}$ & $72.11 \pm 7.14^{\mathrm{a}}$ & $79.21 \pm 4.37^{\mathrm{ab}}$ & $77.74 \pm 8.65^{b}$ & $p \leq 0.001$ \\
\hline Maximum IOP ${ }^{1}$ & $35.56 \pm 9.74^{\mathrm{ab}}$ & $34.16 \pm 13.79^{\mathrm{a}}$ & $24.50 \pm 7.49^{b}$ & $18.28 \pm 2.76^{\mathrm{ab}}$ & $p \leq 0.05$ \\
\hline $\mathrm{MD}^{1}$ & $-16.24 \pm 9.97^{a}$ & $-14.23 \pm 10.27^{\mathrm{a}}$ & $-12.75 \pm 8.51^{a}$ & $0.16 \pm 096$ & $p>0.05$ \\
\hline
\end{tabular}

${ }^{1}$ Mean \pm standard deviation (SD). IOP intraocular pressure, $M D$ mean defect. Groups in row not sharing the same letter are statistically different at $p \leq 0.05$. $P O A G$ primary open angle glaucoma, $P E X G$ pseudoexfoliation glaucoma, $P A C G$ primary angle closure glaucoma 
3. Glaucomatous neuropathy

4. BCVA better than 0.1 (measured using Snellen charts)

5. Senile cataract with BCVA worse than 0.6

6. No ocular diseases except for cataract and glaucoma

7. No previous intraocular surgeries (except for laser iridotomy in PACG patients)

8. No diabetes mellitus

Patients with glaucoma additionally had visual field examination (24-2 strategy on Humphrey Perimeter). Glaucoma was diagnosed based upon the clinical determination of glaucomatous ONH damage (localised or diffuse neuroretinal rim thinning, rim notching, excavation, and/or retinal nerve fiber layer defect) associated with typical, reproducible standard automated perimetry defects. Glaucomatous defect on standard automated perimetry was defined based upon a glaucoma hemifield test result outside normal limits and the presence of at least 3 contiguous test points within the same hemifield on the pattern deviation plot at $P<1 \%$, with at least 1 point at $P<0.5 \%$, on at least 2 consecutive tests, with reliability indices better than $15 \%$.

POAG was diagnosed when wide open-angle (grade III/ IV according to Schaffer's classification) was observed on gonioscopy without the features of any secondary glaucoma. PACG was diagnosed in patients with documented primary angle closure in their medical record who had undergone laser peripheral iridotomy. PEXG was diagnosed when glaucomatous optic neuropathy was accompanied by the presence of dandruff-like pseudoexfoliation material on the anterior lens capsule.

AH samples $(\sim 100 \mu \mathrm{l})$ were obtained from the anterior chamber by an experienced surgeon (TZ) at the beginning of the cataract surgery with a special care to avoid contamination with blood or tears. MiRNA was immediately isolated from AH samples using TaqMan MicroRNA Cells-to-CT Kit (Ambion, Austin, TX, USA) according to the manufacturer's instructions. The isolated miRNA was stored at $-80{ }^{\circ} \mathrm{C}$ for further analysis.

The absorbance of miRNA was measured using a NanoDrop 2000c spectrophotometer (Thermo Fisher Scientific, Waltham, MA, USA), which allowed the authors to perform qualitative and quantitative evaluation. The amount of miRNA per sample in all cases was in the range of 1-350 ng. In addition, RNA analysis was performed using the Agilent Bianalyzer 2100 (Agilent Technologies, Lithuania) and Pico RNA Kit according to the manufacturer's procedure (Supplementary Fig. 1).

Twenty-two miRNAs were selected for analysis on the basis of previous publications $[19,20]$, which showed them to be the most abundant in AH. The list of selected miRNAs was provided in Table 2 .

The reverse transcription (RT) reaction was performed using a set of Custom RT Primer Pools and TaqMan
MicroRNA Reverse Transcription Kit (both Applied Biosystems, Foster, CA, USA) according to the manufacturer's protocol. In the next step, the preamplification reaction was performed using the TaqMan PreAmp Master Mix and Custom PreAmp Primer Pool (both Applied Biosystems) according to the manufacturer's protocol.

The 7900HT Fast Real-Time PCR System and Custom TaqMan Array MircoRNA Card (both Applied Biosystems) were used to analyze the levels of selected miRNAs expression in the tested samples. U6 snRNA was used as the endogenous control. Relative miRNA expression was calculated using the $2^{-\Delta \Delta \mathrm{CT}}$ method [7] in the ExpressionSuite Software v1.03 implemented in the real-time PCR platform. The miRNA was assessed as "Detected" when the expression of a given miRNA was observed in the tested material. Cutoff for $\mathrm{Ct}$ numbers was 40 in ExpressionSuite Software.

Statistical analysis was performed using Statistica 13.5 (StatSoft Polska, Cracow, Poland) and Graph Pad Prism 7 (Graph Pad Software, San Diego, CA, USA), and a $p$ value less than 0.05 was considered significant. Qualitative data were presented as numbers and percentages of the sample. The Chi-squared test $\left(\chi^{2}\right)$ was used to compare the relationships between qualitative variables.

As the data were not normally distributed (Shapiro-Wilk test), Mann-Whitney and Kruskall-Wallis tests with subsequent post hoc analyses were used to compare the levels of miRNA expression between PACG, PEXG, and POAG. In addition, Spearman correlations were performed to examine the relationship between miRNA expression and selected clinical data. Data were expressed as mean with standard deviation (SD). Results were considered statistically significant when $p \leq 0.05$.

Target annotation analysis and network visualization were performed using $\mathrm{R}$ environment (version 3.6.3, https://www.r-project.org) and appropriate packages according to corresponding reference manuals. Identification of miRNA/gene regulatory interactions was performed in silico between selected miRNAs and glaucomaassociated genes harvested from DisGeNET 7.0 database (https://www.disgenet.org/) [21-23], using multiMiR 1.10.0 package (https://bioconductor.org/packages/relea se/bioc/html/multiMiR.html, database version: 2.3.0, updated in 2020-04-15) [24]. Analysis included identification of both experimentally validated (miRecords, miRTarBase, and TarBase databases) and predicted (DIANAmicroT-CDS, ElMMo, MicroCosm, miRanda, miRDB, PicTar, PITA, and TargetScan databases) miRNA/gene interactions.

Visualization of the regulatory network with obtained interactions was performed using Cytoscape v3.7.0 software (https://cytoscape.org/) [25]. 
Table 2 List of miRNAs examined in the study

\begin{tabular}{|c|c|c|c|c|}
\hline Assay ID & Assay name & miRBase ID $^{1}$ & miRBase Accesion Number ${ }^{1}$ & Mature miRNA Sequence $^{1}$ \\
\hline 464340_mat & hsa-miR-4433 & hsa-miR-4433a-3p & MIMAT0018949 & ACAGGAGUGGGGGUGGGACAU \\
\hline 002,362 & hsa-miR-202* & hsa-miR-202-5p & MIMAT0002810 & UUCCUAUGCAUAUACUUCUUUG \\
\hline 463784_mat & hsa-miR-4725-3p & hsa-miR-4725-3p & MIMAT0019844 & UGGGGAAGGCGUCAGUGUCGGG \\
\hline 002,404 & hsa-let-7b* & hsa-let-7b-3p & MIMAT0004482 & CUAUACAACCUACUGCCUUCCC \\
\hline 000,485 & hsa-miR-184 & hsa-miR-184 & MIMAT0000454 & UGGACGGAGAACUGAUAAGGGU \\
\hline 002,858 & hsa-miR-1202 & hsa-miR-1202 & MIMAT0005865 & GUGCCAGCUGCAGUGGGGGAG \\
\hline 000,399 & hsa-miR-23a & hsa-miR-23a-3p & MIMAT0000078 & AUCACAUUGCCAGGGAUUUCC \\
\hline 465775_mat & hsa-miR-3663-3p & hsa-miR-3663-3p & MIMAT0018085 & UGAGCACCACACAGGCCGGGCGC \\
\hline 474380_mat & hsa-miR-6722-3p & hsa-miR-6722-3p & MIMAT0025854 & UGCAGGGGUCGGGUGGGCCAGG \\
\hline 000,449 & hsa-miR-125b & hsa-miR-125b-5p & MIMAT0000423 & UCCCUGAGACCCUAACUUGUGA \\
\hline 471487_mat & hsa-miR-6515-3p & hsa-miR-6515-3p & MIMAT0025487 & UCUCUUCAUCUACCCCCCAG \\
\hline 002,740 & hsa-miR-187* & hsa-miR-187-5p & MIMAT0004561 & GGCUACAACACAGGACCCGGGC \\
\hline 242525_mat & hsa-miR-1260b & hsa-miR-1260b & MIMAT0015041 & AUCCCACCACUGCCACCAU \\
\hline 242546_mat & hsa-miR-3197 & hsa-miR-3197 & MIMAT0015082 & GGAGGCGCAGGCUCGGAAAGGCG \\
\hline 476356_mat & hsa-miR-5001-5p & hsa-miR-5001-5p & MIMAT0021021 & AGGGCUGGACUCAGCGGCGGAGCU \\
\hline 476806_mat & hsa-miR-6132 & hsa-miR-6132 & MIMAT0024616 & AGCAGGGCUGGGGAUUGCAG \\
\hline 463820_mat & hsa-miR-4749-5p & hsa-miR-4749-5p & MIMAT0019885 & UGCGGGGACAGGCCAGGGCAUC \\
\hline 475810_mat & hsa-miR-6717-5p & hsa-miR-6717-5p & MIMAT0025846 & AGGCGAUGUGGGGAUGUAGAGA \\
\hline 462391_mat & hsa-miR-3940-5p & hsa-miR-3940-5p & MIMAT0019229 & GUGGGUUGGGGCGGGCUCUG \\
\hline 464264_mat & hsa-miR-4484 & hsa-miR-4484 & MIMAT0019018 & AAAAGGCGGGAGAAGCCCCA \\
\hline 463950_mat & hsa-miR-4467 & hsa-miR-4467 & MIMAT0018994 & UGGCGGCGGUAGUUAUGGGCUU \\
\hline Hs04258334_pri & hsa-mir-4634 & hsa-mir-4634 & $\begin{array}{l}\text { MI0017261 (stem loop } \\
\text { sequence) }\end{array}$ & $\begin{array}{l}\text { GGACAAGGGCGGCGCGACCGGCC CGG } \\
\text { GGCUCUUGGGCGGCCGCGU UUCCCC } \\
\text { UCC }\end{array}$ \\
\hline 001,973 & U6 snRNA & $\begin{array}{l}\text { NCBI Accession Num- } \\
\text { ber: NR_004394 }\end{array}$ & $\begin{array}{l}\text { Name: RNA, U6 small } \\
\text { nuclear 1, Symbol: RNU6- } \\
1\end{array}$ & $\begin{array}{l}\text { GTGCTCGCTTCGGCAGCACATATA CTA } \\
\text { AAATTGGAACGATACAGAGAA GAT } \\
\text { TAGCATGGCCCCTGCGCAAGG ATG } \\
\text { ACACGCAAATTCGTGAAGCGTT CCA } \\
\text { TATTTT }\end{array}$ \\
\hline
\end{tabular}

${ }^{1}$ According to miRBase 22 (http://www.mirbase.org/)

Functional analysis of target genes was performed using Database for Annotation, Visualization and Integrated Discovery (DAVID) 6.8 tool (https://david.ncifcrf.gov/) [26, 27]. Default Homo sapiens genome was used as a background. Terms of Kyoto Encyclopedia of Genes and Genomes (KEGG), Reactome, and Gene Ontology (GO) categories were searched.

\section{Results}

The ability to detect the expression of selected miRNAs in AH differed in studied glaucoma subgroups (Fig. 1, Table 3). Eight miRNAs were detected in at least $20 \%$ of samples in at least one of the studied groups. Three miRNAs (hsamiR-1202, hsa-miR-1260b, hsa-mir-4634) were detected in at least $20 \%$ of samples in all studied groups (Fig. 1, Supplementary Table 1). The most frequently expressed miRNA in the studied panel was hsa-miR-1260b, which was detected in 19 POAG (100\%), 13 PEXG (92\%), and 7 PACG (77\%) samples (Table 3, Supplementary Table 1).

Seven out of eight the most frequently expressed miRNAs were detected in minimum 3 samples of two glaucoma subgroups and cataract group, enabling differential expression analysis (Table 3, Supplementary Table 2). We did not observe any significant differences in the frequency of expression and the level of expression for these miRNAs; however, there was a tendency for different expressions of hsa-miR-184, -1206b, and -6722-3p between various glaucoma types (Table 1, Fig. 2). Hsa-miR-184 (PEXG 50\%, PACG 22\%, POAG 16\%, $p=0.0885)$ and hsa-miR-6722-3p (PEXG 43\%, PACG 11\%, POAG 16\%, p=0.1180) tended to express more frequently in PEXG patients (Table 3, Fig. 2).

We performed the correlation analysis between expression levels of seven of the most abundantly expressed miRNAs and the clinical status of the patients from the studied groups (Table 4). Interestingly, the expression of both hsa-miR-1260b (moderate correlation, $R=0.47$ ) and 


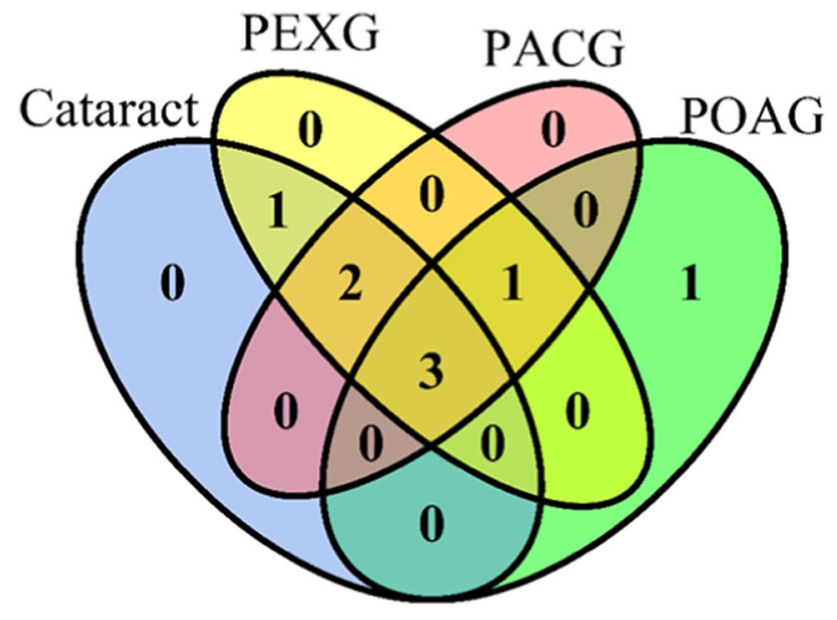

Fig. 1 Venn diagram for miRNAs detected in at least 20\% of samples within cataract, primary open angle glaucoma (POAG), pseudoexfoliation glaucoma (PEXG), and primary angle closure glaucoma (PACG) groups. Only in PAOG is hsa-miR-23a, common for only cataract and PEXG is hsa-miR-6722-3p, common for only PEXG, PACG, and POAG is hsa-miR-6515-3p, common for only cataract, PEXG and PACG are hsa-miR-184 and hsa-miR-187-5p, common for all groups are hsa-miR-1202, hsa-miR-1260b, and hsa-mir-4634. The plot was generated using VennDiagram 1.6.20 package in $\mathrm{R}$

hsa-miR-6515-3p (very strong correlation, $\mathrm{R}=0.89$ ) was statistically significantly correlated with age in POAG. The expression of hsa-miR-1260b was also correlated with the level of maximum IOP in the PACG group (very strong correlation, $\mathrm{R}=0.89$ ). This correlation was no significant in PEXG group, despite the similar level of IOP and hsamiR-1260b expression. However, in the PEXG group, the expression levels of hsa-miR-1260b were correlated with the stage of glaucoma assessed as mean defect (MD) index in visual field (VF) examination (very strong correlation; $p=0.85$ ). As MD is a negative value, it means that with a more advanced stage of glaucoma, the level of hsa-miR$1260 \mathrm{~b}$ tended to decrease.

To recognize a regulatory function of analyzed miRNAs in glaucoma pathology, we performed in silico target annotation analysis between seven the most frequently expressed miRNAs found in $\mathrm{AH}$ of patients with glaucoma (presented in Table 1) and 770 glaucoma-associated genes received from DisGeNET 7.0 database (Concept Unique Identifier "C0017601" was queried). Target annotation analysis requires mature miRNA IDs as input; therefore in the case of hsa-mir-4634 stem-loop miRNA, we used its-derived mature miRNA ID: hsa-miR-4634. Target annotation analysis revealed 69 validated miRNA:gene pairs (Supplementary Table 3) as well as 86 top 10\% predicted miRNA:gene pairs obtained with the highest probability (Supplementary Table 4). Identified interactions were visualized on the regulatory network (Fig. 3).

To indicate biological processes in which miRNA-regulated genes are involved, functional analysis was performed for 124 networked genes using DAVID website tool. Figure 4 presents top ten the most enriched terms of GO (Gene Ontology) Biological Processing, GO Cellular Compartment, GO Molecular Function, KEGG (Kyoto Encyclopedia of Genes and Genomes), and Reactome categories.

Genes found as targets of the most frequently expressed miRNAs in glaucoma were associated with the cardiovascular system development, response to hypoxia, apoptosis, cytoplasm compartment, enzymatic activity, cancer, FoxO, PI3K-AKT and HIF-1 signalling pathways, viral infections, blood coagulation cascade, and regulation of inflammation.

\section{Discussion}

Extracellular miRNAs, both secreted by intraocular cells and derived from blood plasma, play essential role in ocular development and retinal homeostasis [28]. The level of miRNAs in AH was shown to be influenced by damages in the anterior and posterior ocular segment [4]. Moreover, trabecular meshwork cell contractility and extracellular matrix turnover are influenced by distinct miRNAs [29-31] that may therefore be relevant in glaucoma. In the current study, expression of 18 selected miRNA was profiled in $\mathrm{AH}$ of

Table 3 The frequency and the level of expression of the studied miRNAs, which expression enabled statistical analysis

\begin{tabular}{|c|c|c|c|c|c|c|c|c|c|c|c|}
\hline \multirow[t]{2}{*}{ miRNA ID } & \multicolumn{2}{|c|}{ POAG } & \multicolumn{2}{|c|}{ PEXG } & \multicolumn{2}{|c|}{ PACG } & \multicolumn{3}{|c|}{ Statistical analysis } & \multicolumn{2}{|c|}{$\mathrm{POAG}+\mathrm{PEXG}+\mathrm{PACG}$} \\
\hline & $\mathrm{n}$ & mean $\pm \mathrm{SD}$ & $\mathrm{n}$ & mean $\pm \mathrm{SD}$ & $\mathrm{n}$ & mean $\pm \mathrm{SD}$ & $\mathrm{p}^{1}$ & $\mathrm{p}^{2}$ & $\mathrm{p}^{3}$ & $\mathrm{n}$ & mean $\pm \mathrm{SD}$ \\
\hline hsa-miR-1202 & 4 & $-0.44 \pm 1.07$ & 6 & $0.30 \pm 3.10$ & 2 & $-0.47 \pm 0.79$ & $>0.05$ & $>0.05$ & $>0.05$ & 12 & $-0.08 \pm 2.21$ \\
\hline hsa-miR-1260b & 19 & $0.12 \pm 0.79$ & 13 & $-0.16 \pm 0.65$ & 7 & $-0.16 \pm 0.51$ & $>0.05$ & $>0.05$ & $>0.05$ & 39 & $-0.06 \pm 0.71$ \\
\hline hsa-miR-184 & 3 & $0.65 \pm 0.55$ & 7 & $0.44 \pm 0.80$ & 2 & $-0.03 \pm 0.89$ & $>0.05$ & $>0.05$ & $>0.05$ & 12 & $0.41 \pm 0.73$ \\
\hline hsa-miR-187-5p & 3 & $2.45 \pm 5.07$ & 3 & $-1.19 \pm 0.11$ & 2 & $-0.30 \pm 2.98$ & $>0.05$ & $>0.05$ & $>0.05$ & 8 & $0.92 \pm 3.40$ \\
\hline hsa-mir-4634 & 12 & $-0.09 \pm 0.66$ & 7 & $-0.20 \pm 0.50$ & 4 & $-0.16 \pm 0.15$ & $>0.05$ & $>0.05$ & $>0.05$ & 23 & $-0.14 \pm 0.54$ \\
\hline hsa-miR-6515-3p & 8 & $-2.27 \pm 0.90$ & 3 & $0.21 \pm 3.27$ & 2 & $-1.09 \pm 0.52$ & $>0.05$ & $>0.05$ & $>0.05$ & 13 & $-1.52 \pm 1.86$ \\
\hline hsa-miR-6722-3p & 3 & $0.93 \pm 0.79$ & 6 & $0.65 \pm 2.25$ & 1 & $-0.71 \pm \mathrm{N} / \mathrm{A}$ & $>0.05$ & $>0.05$ & $>0.05$ & 10 & $0.60 \pm 1.79$ \\
\hline
\end{tabular}

${ }^{1}$ Statistical significance of expression level between POAG, PEXG, PACG. ${ }^{2}$ Statistical significance of expression frequency. ${ }^{3}$ Statistical significance of expression level between control group and POAG + PEXG + PACG. SD standard deviation, POAG primary open angle glaucoma, $P E X G$ pseudoexfoliation glaucoma, $P A C G$ primary angle closure glaucoma 
Fig. 2 The expression levels of the most frequently detected miRNAs in the studied groups. Data are shown as mean \pm standard deviation (SD). $P O A G$ primary open angle glaucoma, $P E X G$ pseudoexfoliation glaucoma, $P A C G$ primary angle closure glaucoma
hsa-miR-1202

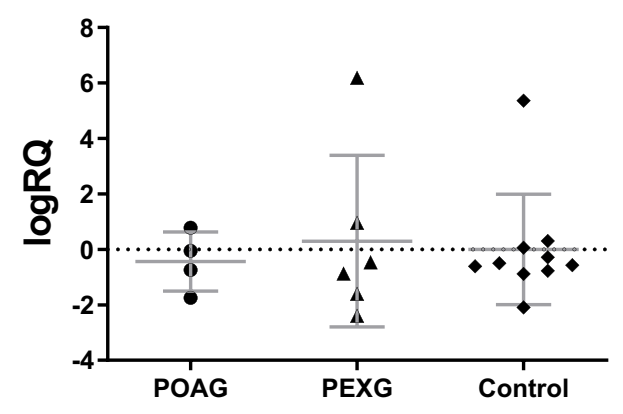

hsa-miR-184

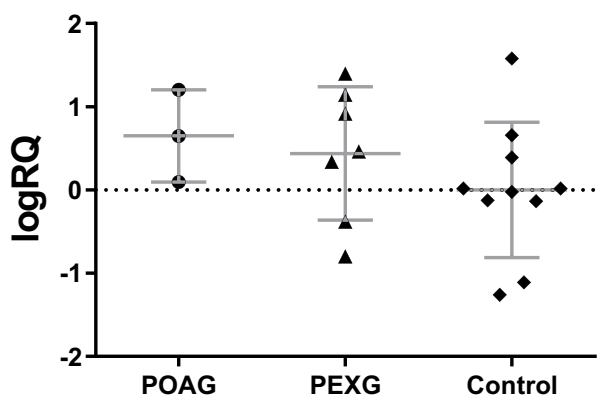

hsa-miR-4634

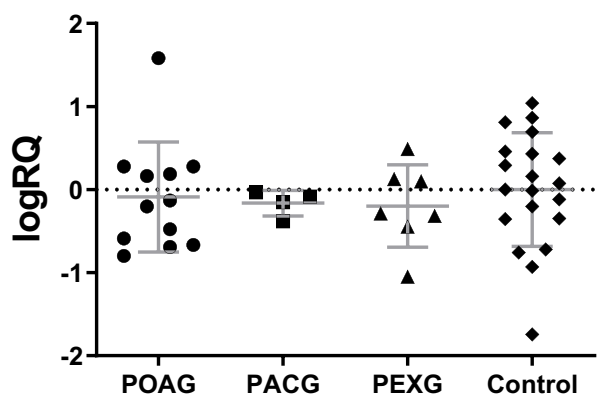

hsa-miR-6722-3p

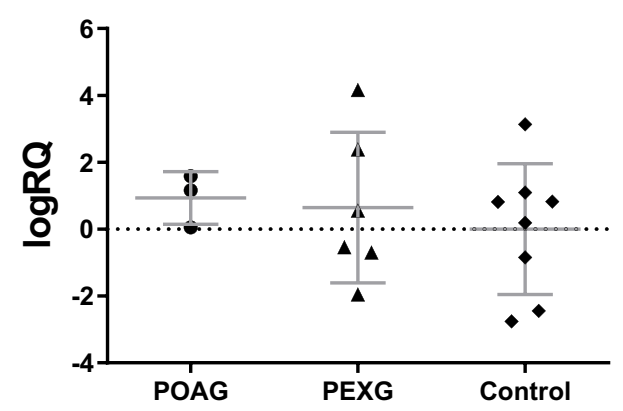

hsa-miR-1260b

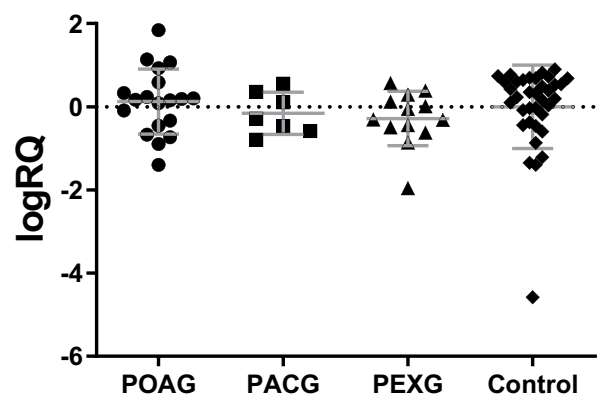

hsa-miR-187-5p

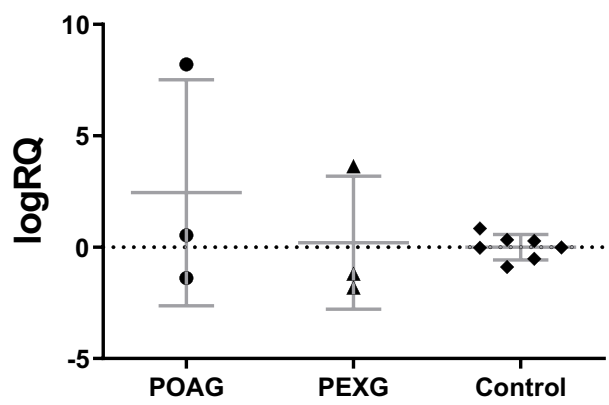

hsa-miR-6515-3p

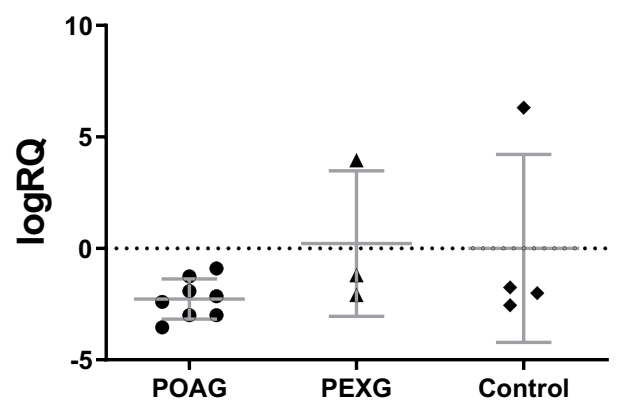

patients with different types of glaucoma (POAG, PACG and PEXG) as well as in cataract group as control to check whether the expression patterns of miRNA may reflect different mechanisms causing IOP elevation.

In our study, seven miRNAs were revealed as the most abundant in AH of glaucoma patients (Table 1, Fig. 2).
However, expression of these miRNAs did not significantly differentiate glaucoma and cataract patients as well as glaucoma subgroups. Our findings are consistent with previous studies, where any of these seven miRNAs were not reported as differentially expressed with statistical significance $[4,32,33]$. 
Table 4 Correlations of miRNA expression levels with age, maximum IOP and MD

\begin{tabular}{|c|c|c|c|c|}
\hline miRNA & Group & Age & Maximum IOP & MD \\
\hline \multirow[t]{4}{*}{ hsa-miR-1260b } & POAG & $0.47 *$ & 0.16 & -0.01 \\
\hline & PACG & -0.38 & $0.86^{*}$ & 0.32 \\
\hline & PEXG & -0.19 & -0.21 & $0.85^{* * * *}$ \\
\hline & Control & 0.05 & 0.36 & $0.48 *$ \\
\hline \multirow[t]{4}{*}{ hsa-miR-6515-3p } & POAG & $0.89 * *$ & 0.16 & -0.07 \\
\hline & PACG & N/A & N/A & N/A \\
\hline & PEXG & 1.00 & 0.50 & -1.00 \\
\hline & Control & -0.20 & 0.47 & -0.95 \\
\hline \multirow[t]{4}{*}{ hsa-miR-4634 } & POAG & 0.40 & 0.07 & -0.38 \\
\hline & PACG & 0.40 & 0.40 & 0.40 \\
\hline & PEXG & -0.36 & 0.38 & 0.29 \\
\hline & Control & 0.26 & $0.58 *$ & 0.01 \\
\hline \multirow[t]{4}{*}{ hsa-miR-1202 } & POAG & -0.63 & -0.40 & 0.40 \\
\hline & PACG & N/A & N/A & N/A \\
\hline & PEXG & -0.26 & 0.23 & 0.20 \\
\hline & Control & 0.36 & $-0.84 *$ & 0.87 \\
\hline \multirow[t]{4}{*}{ hsa-miR-184 } & POAG & 0.50 & 0.50 & 1.00 \\
\hline & PACG & N/A & N/A & N/A \\
\hline & PEXG & -0.33 & 0.22 & 0.57 \\
\hline & Control & -0.36 & 0.37 & -0.27 \\
\hline \multirow[t]{4}{*}{ hsa-miR-6722-3p } & POAG & 1.00 & 0.50 & -0.50 \\
\hline & PACG & N/A & N/A & N/A \\
\hline & PEXG & 0.61 & -0.23 & -0.43 \\
\hline & Control & $-0.95^{*}$ & 0.63 & -0.40 \\
\hline \multirow[t]{4}{*}{ hsa-miR-187 } & POAG & -0.50 & -1.00 & -0.50 \\
\hline & PACG & N/A & N/A & N/A \\
\hline & PEXG & -0.50 & 0.00 & -0.50 \\
\hline & Control & 0.20 & 0.63 & -0.50 \\
\hline
\end{tabular}

${ }^{*} p \leq 0.05$;** $p \leq 0.01 ; * * * p \leq 0.001 ;$ IOP intraocular pressure, $M D$ mean defect, $N / A$ not calculated due to low frequency of detection, $P O A G$ primary open angle glaucoma, $P E X G$ pseudoexfoliation glaucoma, $P A C G$ primary angle closure glaucoma

In our research, the most frequently detected miRNAs in AH of glaucoma patients were hsa-miR-4634 and hsamiR-1260b (Table 1, Fig. 2). Hsa-miR-4634 is a validated regulator of VAV3 (Fig. 3), whose deficiency in mice was associated with an ocular phenotype similar to glaucoma, including elevated IOP, selective loss of retinal ganglion cells and optic nerve head cupping [34]. Hsa-miR-1260b tended to be expressed more frequently in POAG patients. This miRNA was previously shown to target genes regulating proliferation and differentiation of neuronal cells, e.g., LMXIB [35], SMAD4 [36], WNK1 [37], and CREB1 [38]. Genetic variants and mutations in $L M X 1 B$ were previously associated with susceptibility of glaucoma [39-42]. It suggests a possible role of hsa-miR-1260b in glaucoma by controlling cellular differentiation and proliferation; however the mechanism should be further elucidated.
There are data showing the influence of some miRNA variants on glaucoma endophenotype. Ghanbari et al. [43] found that variants in the hsa-miR-612 precursor and in the hsa-miR-4707 seed region were significantly associated with vertical cup-to-disc ratio and cup area. In this study a strong positive correlation between hsa-miR-1260b and MD was observed, which shows that the level of hsa-miR$1260 \mathrm{~b}$ expression decreased in more advanced PEXG cases. It suggests that hsa-miR-1260b may play a protective role in the course of glaucomatous neuropathy. On the other hand, $C R E B$, one of the genes targeted by hsa-miR-1260b, has a neuroprotective effect against hydrogen peroxide-induced retinal ganglion cell death via two downstream cell survival genes, $B D N F$ and BCL2 [44]. Upregulation of $C R E B$ and $B C L 2$ enhances cell survival and reduces apoptosis after optic nerve crush [45] as well as activate MEK/ERK/CREB pathway as a protective mechanism of silibinin against blue light damage of retinal ganglion cells [46]. The effect of interaction between hsa-miR-1260b and CREB should be investigated in further studies.

Hsa-miR-1260b is also an essential regulator of vascular smooth muscle cells proliferation in response to hypoxia [47]. In all areas of the trabecular meshwork and especially in the area external to Schlemm's canal, there are cells that have cytoplasm rich in actin filaments and have characteristics common to smooth muscle cells [48] and thus may play a role in $\mathrm{AH}$ outflow modification. In our study, we observed significant positive correlation between the expression of hsa-miR-1260b and maximum IOP in PACG patients (Table 4), which suggests a probable relation of hsa-miR$1260 \mathrm{~b}$ to mechanisms of IOP increase. However, it may be a part of some PACG-specific pathomechanisms because the IOP level did not differ in patients with PACG and POAG, and in the case of the latter, the correlation between the level of hsa-miR-1260b expression and IOP was not significant.

Additionally, in our research, hsa-miR-1260b was found to be a validated target of SMAD4 and SFRPI (Fig. 3), which are involved in the outflow regulatory mechanisms in eye anterior chamber. These two genes participate in the inhibitory crosstalk between TGF $\beta /$ Smad and Wnt signaling pathways, previously described in human glaucomatous trabecular meshwork cells [49]. Activation of TGF $\beta / \mathrm{Smad}$ signaling and upregulation of SMAD4 were reported in oxidative stress treated human trabecular meshwork cells and was associated with increased production of extracellular matrix [50]. Wnt signaling and its key mediator $\beta$-catenin were reported to be inhibited in elevated IOP by upregulation of SFRP1 [49]. Activation of Wnt pathway in glaucoma alleviated detrimental effects of overactivated TGF $\beta / \mathrm{Smad}$ signaling, since Wnt signaling pathway facilitates AH outflow and decreases IOP [51, 52]. Therefore, the protective effect of hsa-miR-1260b could result from inhibition of SMAD4 and SFRPI. 
Fig. 3 Regulatory network presenting interactions between seven most frequently expressed miRNAs in glaucoma and genes obtained from DisGeNET 7.0 database as associated with glaucoma. Interactions were found in silico using multiMiR 1.10.0 package in $\mathrm{R}$

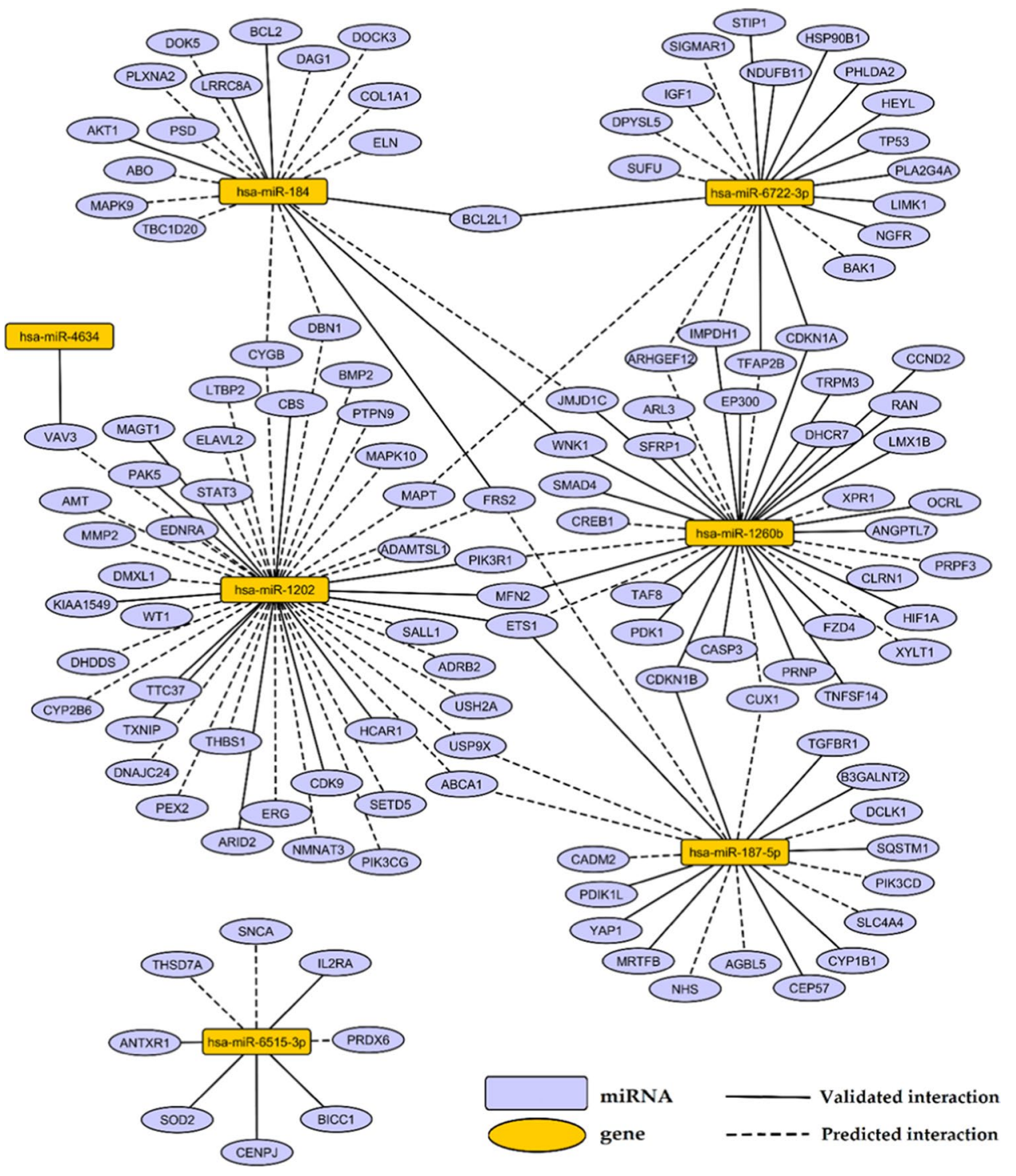

Another miRNA detected in all studied groups is hsamiR-184, which is one of the most abundantly expressed miRNAs in normal human ciliary body, cornea, and trabecular meshwork [53]. Similarly, hsa-miR-184 is also very abundant in $\mathrm{AH}$, with a level much higher than in plasma [54]. This miRNA is also highly expressed in both transparent and cataract lens samples [55]. Prior studies reported high expression of this miRNA in $\mathrm{AH}$ of patients with POAG and cataract, however without statistical significant difference [4], which is in consistence with our results (Table 1). We additionally observed the tendency of hsamiR-184 to be expressed more frequently in AH samples of patients with pseudoexfoliation syndrome, which is the risk factor not only for glaucoma but also for cataract, indicating a potential role of this miRNA in both conditions. Previous studies reported four different point mutations in hsa-miR-184, which were linked with lens/corneal dystrophy and blindness [56-59]; however, function of hsa-miR-184 under homeostasis and its relation to eye pathology remain to be investigated [8].

Apoptosis and dendritic detraction of retinal ganglion cells are main processes contributing to each type of glaucoma [60]. Target annotation analysis performed in silico in our study showed that hsa-miR-184 targets genes controlling apoptosis, including BCL2, BCL2L1, MAPK9, and AKT1 (Fig. 2). Possible repression of these anti-apoptotic genes mediated by interactions with hsa-miR-184 may contribute to potentiate apoptosis potentially aggravating glaucoma outcome; however, this hypothesis requires further verification in experimental studies. Other miRNAs abundantly expressed in $\mathrm{AH}$ of glaucoma patients were also showed in silico in our study as regulators of genes related to apoptosis, e.g., STAT3, BMP2 (targets for hsa-miR-1202), CASP3, $P D K I$ (targets for hsa-miR-1260b), BAK1, and TP53 (targets 


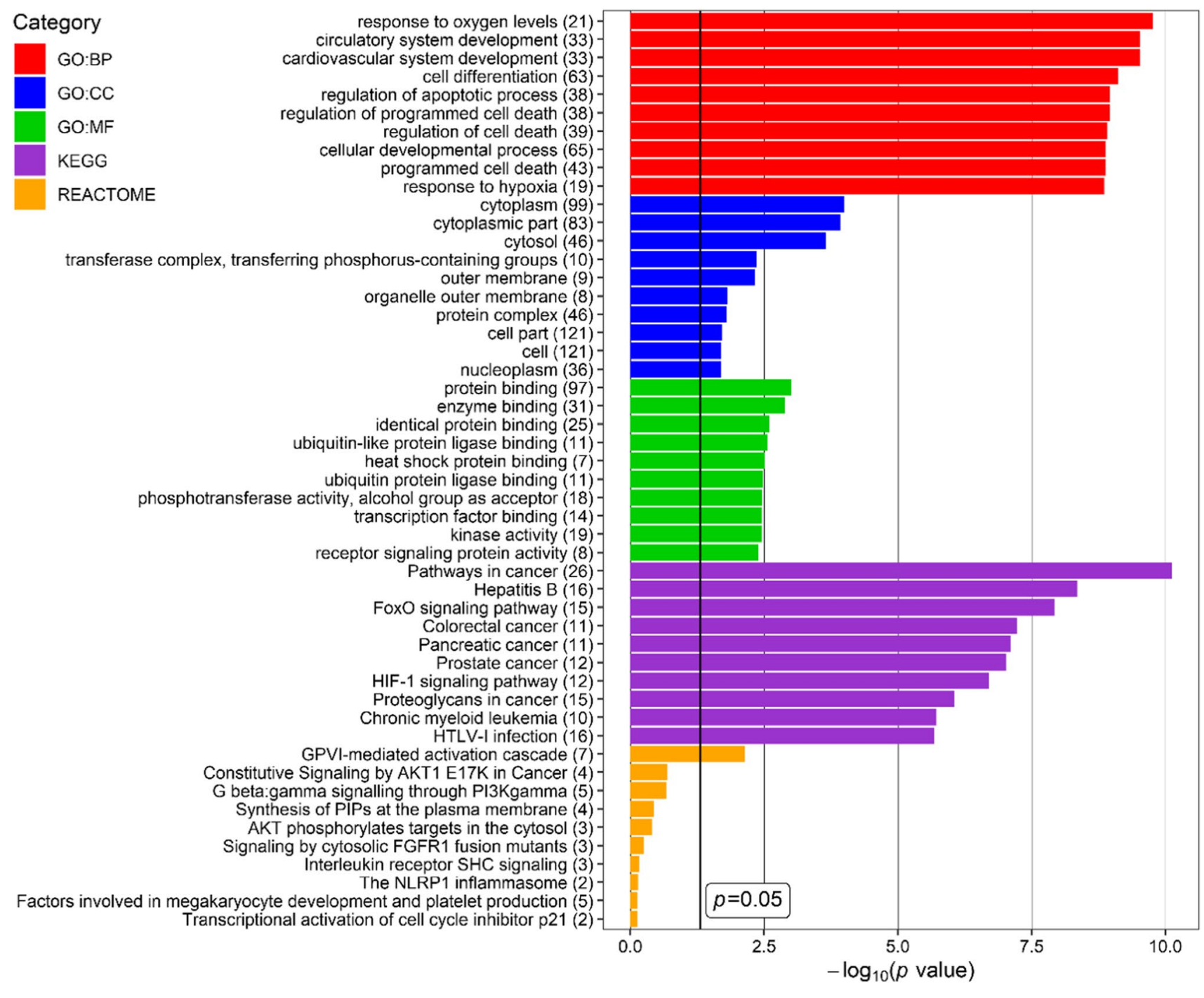

Fig. 4 Top ten the most enriched terms of GO (Gene Ontology) Biological Processing (GO:BP), GO Cellular Compartment (GO:CC), GO Molecular Function (GO:MF), KEGG (Kyoto Encyclopedia of Genes and Genomes) and REACTOME categories, revealed for glaucoma-associated genes targeted by miRNAs found in the current study as the most frequently expressed in aqueous humour $(\mathrm{AH})$

for hsa-miR-6722-3p) (Fig. 2). These findings indicate that miRNAs are involved in regulation of cell apoptosis and survival during glaucoma course and further studies are needed to elucidate this mechanism.

The proper AH flow is maintained by trabecular meshwork cells, which exhibit high susceptibility to injury induced by oxidative free radicals. Dysfunctions of these cells caused by excessive oxygen species may contribute to increase in IOP and glaucoma $[10,11]$. There is a piece of evidence that oxidative stress, together with mitochondrial impairment and pathogenic events, contributes to a complex network of mechanisms leading to glaucoma [12, 13, 61]. Interestingly, target annotation analysis revealed validated of patients with glaucoma. $P$ value - EASE score for enrichment adjusted by Benjamini correction for multiple hypothesis testing. The number in brackets following name of terms indicates number of associated genes. The plot was generated using ggplot2 3.3.0 package in $\mathrm{R}$

interactions of hsa-miR-6515-3p with eight genes (Fig. 2) and two out of them, PRDX6 and SOD2, were previously associated with response to oxidative stress, which is a major cause of glaucoma pathogenesis [62]. PRDX6 was previously shown to delay senescence and limit reactive oxygen species accumulation in human trabecular meshwork cells, and its downregulation was observed in aged and glaucomatous trabecular meshwork cells [63]. SOD2 encodes the manganesedependent superoxide dismutase that acts as a mitochondrial antioxidant enzyme [64], and higher level of SOD enzyme was evidenced in $\mathrm{AH}$ of POAG patients in comparison to cataract subjects [65]. Targeting of PRDX6 and SOD2 by hsa-miR-6515-3p could be a possible mechanism promoting 
cellular senescence and impairing antioxidant ability, thus also contributing to glaucoma. Additionally, many polymorphisms identified in SOD2 and PRDX6 genes [66-68] may potentially influenced interactions with miRNAs affecting susceptibility to glaucoma.

Wang et al. [9] demonstrated that miRNAs could be oxidised in response to oxidative stress. Upon oxidative modification, hsa-miR-184 misrecognizes mRNA for Bcl-xL and $\mathrm{Bcl}-\mathrm{w}$, which are not its native targets, resulting in initiation of cellular apoptosis. Moreover, Gartaganis et al. [14] found that in PEX syndrome, AH samples showed a decrease in glutathione concentration and an increase in glutathione disulfide level, which suggests that oxidation stress plays a role in the PEX pathogenesis and progression. Reduced levels of selenium in $\mathrm{AH}$, conjunctival specimens, and serum of PEX patients also support the hypothesis that the impairment of the antioxidant defense system participates in PEX pathogenesis [69]. Thus, a higher frequency of hsa-miR-184 expression in the PEXG group observed in this study may support previous results.

In glaucoma pathogenesis, blood flow reduction and ischemia are postulated as possible causative mechanisms of optic nerve neuropathy. Reperfusion injury, one of proposed mechanisms, refers to damage to nerve tissue caused when blood supply improves to the tissue after a period of ischemia. The absence of oxygen and nutrients from tissue creates a condition in which the restoration of circulation results in inflammation and oxidative damage rather than restoration of normal function [70]. One of the most enriched functional terms of genes targeted by selected seven the most frequently expressed miRNAs in $\mathrm{AH}$ of patients with glaucoma was "response to oxygen levels" and "response to hypoxia" (Fig. 3). Our in silico target annotation analysis showed that hsa-miR-1260b, hsa-miR-1202, and hsa-miR-187-5p target ETS1 (Fig. 2), which is a transcription factor undergoing upregulation under hypoxia condition and participate in induction of hypoxia-inducible genes expression [71]. Besides ETS1, hsa-miR-1260b targets other hypoxia-associated genes, like HIFl and PDK1 [72] (Fig. 2). The potential role of this miRNA in response to hypoxia in glaucoma neuropathy should be further elucidated.

Apart from elevated IOP, the major risk factor for POAG is older age [73]. In this study, we observed a correlation between age and expression of both hsa-miR6515-3p and hsa-miR-1260b in POAG patients, which may show that these miRNAs regulate aging-related processes contributing to glaucoma pathogenesis. An association between glaucoma and neurodegenerative diseases of the central nervous system, such as Alzheimer's disease (AD), age-related dementia with progressive deterioration of memory and cognition, has also been reported [74]. In our study, hsa-miR-184 and hsa-miR-6722-3p tended to be expressed more frequently in PEXG patients when compared with POAG and PACG groups (Fig. 2) and with the mean level of expression slightly lower than in POAG (difference not significant). Kumar et al. [15] found that miR-6722 was downregulated in serum of patients with AD. Decrease in expression of MiR-184 was found in hippocampus of patients with late-onset $\mathrm{AD}$ [75]. The major pathological hallmarks of AD include accumulation of large extracellular $\beta$-amyloid plaques and intracellular fibrillary tangles of abnormally phosphorylated tau protein. The deposition of amyloid-like material in PEX syndrome shares some features with the findings in AD [16]. Amyloid- $\beta$ peptide has also been demonstrated in AH of PEX patients [17]. In keeping with these observations, a possible relationship between PEX and AD has been suggested, which is consistent with our results. Additionally, Inoue et al. [18] found that PEXG and POAG patients manifested elevated levels of some AD biomarkers (apolipoprotein E, transthyretin) in $\mathrm{AH}$, and this level was related to the severity of glaucoma.

This study has some limitations. First, the control group constitutes of patients with senile cataract without glaucoma. The impact of cataract in glaucoma subgroups on miRNA expression should be taken into consideration, as part of the dysregulated miRNAs found in previous studies may be related to both cataract and glaucoma [4]. Moreover, the number of evaluated samples was not equal for all studied groups. It partially reflects the incidence rate of glaucoma in our population and is also connected with the technical difficulty of obtaining the AH samples from the patients with shallow anterior chamber (PACG). Finally, the study evaluates only the group of miRNAs selected after the careful reviewing of the publications available at the time of the study design $[19,20]$ concerning the subject at the time of study design. The authors decided to select the miRNA most frequently expressed, according to these publications, in the aqueous humor. Although this was the only possible strategy, the authors are aware of the possible bias caused by tightening of the studied panel.

To sum up, in this study we were able to show the expression of selected miRNAs in AH of glaucoma patients. The level of expression was similar for various glaucoma types, but the frequency of expression tended to differ. Additionally, some correlations between clinical features and the studied miRNAs were found, which confirms the possibility that miRNAs influence glaucoma endophenotype. Potential functions of genes targeted by the most frequently detected miRNAs were presented, indicating the important role of miRNAs in glaucoma pathology.

Supplementary Information The online version contains supplementary material available at https://doi.org/10.1007/s00417-021-05214-z. 
Authors' contributions Conceptualization, E.K.-J.; methodology, E.K.J.; software, M.C., D.Z.; formal analysis, M.C.; investigation, E.K.-J., M.C., K.G., D.Z, and D.W.-D.; data curation, M.C.; writing-original draft preparation, E.K.-J., M.C., D.Z., and D.W.-D.; writing-review and editing, E.K.-J. and K.G.; supervision, A.B.-K., J.K., and T.Ż.

Data availability All data and materials support our published claims and comply with field standards.

Code availability Not applicable.

\section{Declarations}

Ethics approval Ethics approval for this study was obtained from the Ethics Committee of Medical University of Lublin (approval No. KE-0254/107/2020).

Consent to participate Patient consent form was obtained.

Consent for publication All authors have read and agreed to the published version of the manuscript.

Conflict of interest The authors declare no conflict of interest.

Open Access This article is licensed under a Creative Commons Attribution 4.0 International License, which permits use, sharing, adaptation, distribution and reproduction in any medium or format, as long as you give appropriate credit to the original author(s) and the source, provide a link to the Creative Commons licence, and indicate if changes were made. The images or other third party material in this article are included in the article's Creative Commons licence, unless indicated otherwise in a credit line to the material. If material is not included in the article's Creative Commons licence and your intended use is not permitted by statutory regulation or exceeds the permitted use, you will need to obtain permission directly from the copyright holder. To view a copy of this licence, visit http://creativecommons.org/licenses/by/4.0/.

\section{References}

1. Alipoor SD, Mortaz E, Tabarsi P et al (2017) Bovis Bacillus Calmette-Guerin (BCG) infection induces exosomal miRNA release by human macrophages. J Transl Med 15:105

2. Tamm ER, Braunger BM, Fuchshofer R (2015) Intraocular pressure and the mechanisms involved in resistance of the aqueous humor flow in the trabecular meshwork outflow pathways. Prog Mol Biol Transl Sci 134:301-314

3. Guo H, Ingolia NT, Weissman JS, Bartel DP (2010) Mammalian microRNAs predominantly act to decrease target mRNA levels. Nature 466:835-840

4. Liu Y, Chen Y, Wang Y et al (2018) microRNA profiling in glaucoma eyes with varying degrees of optic neuropathy by using next-generation sequencing. Invest Ophthalmol Vis Sci 59:2955-2966

5. Mozaffarieh M, Flammer J (2009) The mechanism of glaucomatous damage to the optic nerve. Eur Ophthal Rev 03:33-35

6. Mitchell PS, Parkin RK, Kroh EM et al (2008) Circulating microRNAs as stable blood-based markers for cancer detection. Proc Natl Acad Sci USA 105:10513-10518
7. Livak KJ, Schmittgen TD (2001) Analysis of relative gene expression data using real-time quantitative PCR and the 2$\Delta \Delta C T$ method. Methods 25:402-408

8. Nagosa S, Leesch F, Putin D et al (2017) microRNA-184 induces a commitment switch to epidermal differentiation. Stem Cell Rep 9:1991-2004

9. Wang J-X, Gao J, Ding S-L et al (2015) Oxidative modification of miR-184 enables it to target Bcl-xL and Bcl-w. Mol Cell 59:50-61

10. Alvarado J, Murphy C, Juster R (1984) Trabecular meshwork cellularity in primary open-angle glaucoma and nonglaucomatous normals. Ophthalmology 91:564-579

11. Izzotti A, Saccà SC, Longobardi M, Cartiglia C (2009) Sensitivity of ocular anterior chamber tissues to oxidative damage and its relevance to the pathogenesis of glaucoma. Invest Ophthalmol Vis Sci 50:5251-5258

12. Izzotti A (2010) Mitochondrial damage in the trabecular meshwork of patients with glaucoma. Arch Ophthalmol 128:724-730

13. Saccà SC, Izzotti A (2014) Focus on molecular events in the anterior chamber leading to glaucoma. Cell Mol Life Sci 71:2197-2218

14. Gartaganis SP, Georgakopoulos CD, Patsoukis NE, Gotsis SS, Gartaganis VS, Georgiou CD (2005) Glutathione and lipid peroxide changes in pseudoexfoliation syndrome. Curr Eye Res 30:647-651

15. Kumar S, Vijayan M, Reddy PH (2017) MicroRNA-455-3p as a potential peripheral biomarker for Alzheimer's disease. Hum Mol Genet 26:3808-3822

16. Schlötzer-Schrehardt U, Naumann GOH (2006) Ocular and systemic pseudoexfoliation syndrome. Am J Ophthalmol 141:921-937

17. Janciauskiene S, Krakau T (2001) Alzheimer's peptide: a possible link between glaucoma, exfoliation syndrome and Alzheimer's disease. Acta Ophthalmol Scand 79:328-329

18. Inoue T, Kawaji T, Tanihara H (2013) Elevated levels of multiple biomarkers of Alzheimer's disease in the aqueous humor of eyes with open-angle glaucoma. Invest Ophthalmol Vis Sci 54:5353-5358

19. Tanaka Y, Tsuda S, Kunikata H et al (2015) Profiles of extracellular miRNAs in the aqueous humor of glaucoma patients assessed with a microarray system. Sci Rep 4:5089

20. Dunmire JJ, Lagouros E, Bouhenni RA, Jones M, Edward DP (2013) MicroRNA in aqueous humor from patients with cataract. Exp Eye Res 108:68-71

21. Piñero J, Ramírez-Anguita JM, Saüch-Pitarch J et al (2019) The DisGeNET knowledge platform for disease genomics: 2019 update. Nucleic Acids Res gkz1021

22. Piñero J, Bravo À, Queralt-Rosinach N et al (2017) DisGeNET: a comprehensive platform integrating information on human disease-associated genes and variants. Nucleic Acids Res 45:D833-D839

23. Pinero J, Queralt-Rosinach N, Bravo A et al (2015) DisGeNET: a discovery platform for the dynamical exploration of human diseases and their genes. Database 2015:bav028-bav028

24. Ru Y, Kechris KJ, Tabakoff B et al (2014) ThemultiMiR R package and database: integration of microRNA-target interactions along with their disease and drug associations. Nucleic Acids Res 42:e133-e133

25. Shannon P, Markiel A, Ozier O et al (2003) Cytoscape: a software environment for integrated models of biomolecular interaction networks. Genome Res 13:2498-2504

26. Huang DW, Sherman BT, Lempicki RA (2009) Systematic and integrative analysis of large gene lists using DAVID bioinformatics resources. Nat Protoc 4:44-57 
27. Huang DW, Sherman BT, Lempicki RA (2009) Bioinformatics enrichment tools: paths toward the comprehensive functional analysis of large gene lists. Nucleic Acids Res 37:1-13

28. Freddo TF (2013) A contemporary concept of the blood-aqueous barrier. Progr Retin Eye Res 32:181-195

29. Luna C, Li G, Qiu J, Epstein DL, Gonzalez P (2011) Crosstalk between miR-29 and Transforming Growth Factor-Betas in Trabecular Meshwork Cells. Invest Ophthalmol Vis Sci 52:3567-3572

30. Luna C, Li G, Huang J et al (2012) Regulation of trabecular meshwork cell contraction and intraocular pressure by miR200c. PLoS ONE 7:e51688

31. Villarreal G, Oh D-J, Kang MH, Rhee DJ (2011) Coordinated Regulation of Extracellular Matrix Synthesis by the MicroRNA-29 Family in the Trabecular Meshwork. Invest Ophthalmol Vis Sci 52:3391-3397

32. Drewry MD, Challa P, Kuchtey JG et al (2018) Differentially expressed microRNAs in the aqueous humor of patients with exfoliation glaucoma or primary open-angle glaucoma. Hum Mol Genet 27:1263-1275

33. Hindle AG, Thoonen R, Jasien JV et al (2019) Identification of Candidate miRNA Biomarkers for Glaucoma. Invest Ophthalmol Vis Sci 60:134-146

34. Fujikawa K, Iwata T, Inoue K et al (2010) VAV2 and VAV3 as candidate disease genes for spontaneous glaucoma in mice and humans. PLoS ONE 5:e9050

35. Yan CH, Levesque M, Claxton S, Johnson RL, Ang S-L (2011) Lmx $1 \mathrm{a}$ and $\operatorname{lm} x 1 \mathrm{~b}$ function cooperatively to regulate proliferation, specification, and differentiation of midbrain dopaminergic progenitors. J Neurosci 31:12413-12425

36. Kawaguchi-Niida M, Shibata N, Furuta Y (2017) Smad4 is essential for directional progression from committed neural progenitor cells through neuronal differentiation in the postnatal mouse brain. Mol Cell Neurosci 83:55-64

37. Sun X, Gao L, Yu RK, Zeng G (2017) Down-regulation of WNK1 protein kinase in neural progenitor cells suppresses cell proliferation and migration. J Neurochem 99:1114-1121

38. Smith DK, Yang J, Liu M-L, Zhang C-L (2016) Small molecules modulate chromatin accessibility to promote NEUROG2-mediated fibroblast-to-neuron reprogramming. Stem Cell Rep 7:955-969

39. Cross SH, Macalinao DG, McKie L et al (2014) A dominant-negative mutation of mouse $1 \mathrm{mx} 1 \mathrm{~b}$ causes glaucoma and is semi-lethal via LBD1-mediated dimerisation. PLoS Genet 10:e1004359

40. Gharahkhani P, Burdon KP, Cooke Bailey JN et al (2018) Analysis combining correlated glaucoma traits identifies five new risk loci for open-angle glaucoma. Sci Rep 8:3124

41. Choquet H, Paylakhi S, Kneeland SC et al (2018) A multiethnic genome-wide association study of primary open-angle glaucoma identifies novel risk loci. Nat Commun 9:2278

42. Shiga Y, Akiyama M, Nishiguchi KM et al (2018) Genome-wide association study identifies seven novel susceptibility loci for primary open-angle glaucoma. Hum Mol Genet 27:1486-1496

43. Ghanbari M, Iglesias AI, Springelkamp H et al (2017) A GenomeWide Scan for MicroRNA-Related Genetic Variants Associated With Primary Open-Angle Glaucoma. Invest Ophthalmol Vis Sci 58:5368-5377

44. Shao Y, Yu Y, Zhou Q, Li C, Yang L, Pei C (2015) Inhibition of miR-134 Protects Against Hydrogen Peroxide-Induced Apoptosis in Retinal Ganglion Cells. J Mol Neurosci 56:461-471

45. Ye D, Shi Y, Xu Y, Huang J (2019) PACAP Attenuates optic nerve crush-induced retinal ganglion cell apoptosis via activation of the CREB-Bcl-2 pathway. J Mol Neurosci 68:475-484

46. Shen Y, Zhao H, Wang Z et al (2019) Silibinin declines blue light-induced apoptosis and inflammation through MEK/ERK/ CREB of retinal ganglion cells. Artif Cells Nanomed Biotechnol 47:4059-4065
47. Seong M, Kang H (2020) Hypoxia-induced miR-1260b regulates vascular smooth muscle cell proliferation by targeting GDF11. BMB Rep 53:206-211

48. Gipson IK, Anderson RA (1979) Actin filaments in cells of human trabecular meshwork and Schlemm's canal. Invest Ophthalmol Vis Sci 18:547-561

49. Webber HC, Bermudez JY, Sethi A, Clark AF, Mao W (2016) Crosstalk between TGF $\beta$ and Wnt signaling pathways in the human trabecular meshwork. Exp Eye Res 148:97-102

50. Shen W, Han Y, Huang B et al (2015) MicroRNA-483-3p Inhibits extracellular matrix production by targeting Smad4 in human trabecular meshwork cells. Invest Ophthalmol Vis Sci 56:8419

51. Wang W-H, McNatt LG, Pang I-H et al (2008) Increased expression of the WNT antagonist sFRP-1 in glaucoma elevates intraocular pressure. J Clin Invest JCI33871

52. Villarreal G, Chatterjee A, Oh SS, Oh D-J, Kang MH, Rhee DJ (2014) Canonical Wnt Signaling Regulates Extracellular Matrix Expression in the Trabecular Meshwork. Invest Ophthalmol Vis Sci 55:7433

53. Drewry M, Helwa I, Allingham RR, Hauser MA, Liu Y (2016) miRNA profile in three different normal human ocular tissues by miRNA-Seq. Invest Ophthalmol Vis Sci 57:3731

54. Wecker T, Hoffmeier K, Plötner A et al (2016) MicroRNA profiling in aqueous humor of individual human eyes by next-generation sequencing. Invest Ophthalmol Vis Sci 57:1706

55. Wu C, Lin H, Wang Q et al (2012) Discrepant expression of MicroRNAs in transparent and cataractous human lenses. Invest Ophthalmol Vis Sci 53:3906

56. Farzadfard A, Nassiri N, Moghadam T, Paylakhi S, Elahi E (2016) Screening for MIR184 mutations in Iranian patients with keratoconus. J Ophthalmic Vis Res 11:3-7

57. Hughes AE, Bradley DT, Campbell M et al (2011) Mutation altering the miR-184 seed Region Causes Familial Keratoconus with Cataract. Am J Hum Genet 89:628-633

58. Lechner J, Bae HA, Guduric-Fuchs J et al (2013) Mutational Analysis of MIR184 in Sporadic Keratoconus and Myopia. Invest Ophthalmol Vis Sci 54:5266-5272

59. Iliff BW, Riazuddin SA, Gottsch JD (2012) A Single-Base Substitution in the Seed Region of miR-184 Causes EDICT Syndrome. Invest Ophthalmol Vis Sci 53:348-353

60. Almasieh M, Wilson AM, Morquette B, Cueva Vargas JL, Di Polo A (2012) The molecular basis of retinal ganglion cell death in glaucoma. Prog Retin Eye Res 31:152-181

61. Evangelho K, Mogilevskaya M, Losada-Barragan M, VargasSanchez JK (2019) Pathophysiology of primary open-angle glaucoma from a neuroinflammatory and neurotoxicity perspective: a review of the literature. Int Ophthalmol 39:259-271

62. Tang B, Li S, Cao W, Sun X (2019) The association of oxidative stress status with open-angle glaucoma and exfoliation glaucoma: a systematic review and meta-analysis. J Ophthalmol 2019:1-14

63. Chhunchha B, Singh P, Stamer WD, Singh DP (2017) Prdx6 retards senescence and restores trabecular meshwork cell health by regulating reactive oxygen species. Cell Death Discov 3:17060

64. Pilar Sosa Idelchik M, Begley U, Begley TJ, Melendez TJ (2017) Mitochondrial ROS control of cancer. Semin Cancer Biol 47:57-66

65. Ghanem AA, Arafa LF, El-Baz A (2010) Oxidative Stress Markers in Patients with Primary Open-Angle Glaucoma. Curr Eye Res 35:295-301

66. Abu-Amero KK, Azad TA, Mousa A, Osman EA, Sultan T, AlObeidan SA (2015) Association of SOD2 Mutation (c.47T > C) with Various Primary Angle Closure Glaucoma Clinical Indices. Ophthalmic Genet 36:180-183

67. Celojevic D, Nilsson S, Kalaboukhova L et al (2014) Genetic variation of superoxide dismutases in patients with primary open-angle glaucoma. Ophthalmic Genet 35:79-84 
68. Seibold P, Hall P, Schoof N et al (2013) Polymorphisms in oxidative stress-related genes and mortality in breast cancer patients-potential differential effects by radiotherapy? Breast 22:817-823

69. Yilmaz A, Ayaz L, Tamer L (2017) Selenium and pseudoexfoliation syndrome. Am J Ophthalmol 151:272-276.e1

70. Flammer J, Mozaffarieh M (2007) What is the present pathogenetic concept of glaucomatous optic neuropathy? Surv Ophthalmol 52:S162-S173

71. Salnikow K, Aprelikova O, Ivanov S et al (2008) Regulation of hypoxia-inducible genes by ETS1 transcription factor. Carcinogenesis 29:1493-1499

72. Xie Y, Shi X, Sheng K et al (2019) PI3K/Akt signaling transduction pathway, erythropoiesis and glycolysis in hypoxia (Review). Mol Med Rep 19:783-791
73. Quigley HA (2011) Glaucoma. Lancet 377:1367-1377

74. Mancino R, Martucci A, Cesareo M et al (2018) Glaucoma and Alzheimer disease: one age-related neurodegenerative disease of the brain. Curr Neuropharmacol 16:971-977

75. Annese A, Manzari C, Lionetti C et al (2018) Whole transcriptome profiling of Late-Onset Alzheimer's disease patients provides insights into the molecular changes involved in the disease. Sci Rep $8: 4282$

Publisher's note Springer Nature remains neutral with regard to jurisdictional claims in published maps and institutional affiliations. 